\title{
Sinhala translation of child behaviour checklist: validity and reliability
}

\author{
B C V Senaratna ${ }^{1}$, H Perera² and P Fonseka ${ }^{3}$
}

\begin{abstract}
Objective To translate the child behaviour checklist (CBCL) into Sinhala and validate it for assessment of mental health status of children aged 5-10 years.

Design and setting Translation/back-translation method was used to translate the English $\mathrm{CBCL}$ into Sinhala. Each item in the Sinhala CBCL (CBCL-S) was rated by mental health professionals to determine semantics, content, and conceptual validity types. To ascertain criterion validity, total scores obtained for CBCL-S by administering it to parents or parent surrogates of 49 girls and 80 boys aged $5-10$ years attending the specialist psychiatry clinics and 69 boys and 69 girls in the same age group from the community were compared with
\end{abstract}

clinical diagnoses by a child psychiatrist. Receiver operator characteristic curves were drawn to obtain the cut-off points in CBCL-S for boys and girls separately.

Results Semantics, content, and conceptual and criterion validity of CBCL-S were satisfactory. At the cut-off level of 39 , CBCL-S had a sensitivity of $90 \%$ and a specificity of $88 \%$ for boys and a sensitivity of $89 \%$ and a specificity of $92 \%$ for girls. Internal consistency, test-retest reliability, and inter-interviewer reliability of CBCL-S were satisfactory.

Interpretation CBCL-S is a valid and reliable instrument to measure mental health status of Sinhalese children aged 5-10 years in Sri Lanka.

${ }^{1}$ Lecturer and ${ }^{3}$ Professor in Community Medicine, Faculty of Medical Sciences, University of Sri Jayewardenepura. ${ }^{2}$ Professor in Psychological Medicine, Faculty of Medicine, University of Colombo.

Correspondence: BCVS, e-mail <chamaravs@yahoo.com>. Received 21 September and accepted 15 December 2007. Conflicts of interests: none declared. 


\section{Introduction}

The Child Behaviour Checklist (CBCL) is a commonly used instrument worldwide to measure the mental health status of children aged 4-18 years [1,2]. Due to its comprehen-siveness in measurement, it is often used as the gold standard for developing other similar assessment tools. CBCL obtains reports from parents or parent surrogates regarding the social and academic competencies of children. CBCL also identifies specific behavioural and emotional problems, current and in the past 6 months, under 8 subscales in a 118 -item questionnaire. These subscales fall under two broad internalising and externalising scales. Withdrawn, somatic complaints and anxious/depressed subscales are included in the internalising scale, and delinquent behaviour and aggressive behaviour subscales form the externalising scale. The remaining subscales of social problems, thought problems, attention problems, and sex problems are considered separate from the two broad scales.

CBCL has been translated in to 78 different languages, including 5 Indian languages and has been used in crosscultural research [1,3]. The English version of CBCL was originally validated in 1989 on a sample of 3000 children, referred and non-referred to mental health services [4]. CBCL and its subscales have been extensively used in research and have shown good validity, reliability, and predictive values $[2,4,5,6-10]$.

Translation and adaptation of such an instrument to a new socio-cultural setting demands an appropriate translation process and establishing measurement properties relevant to the target language [11, 12]. Also, for a translation to be meaningful, the content, semantics and concepts in the instrument have to be suitably adapted to the target culture and language [11, 12, 13, 14]. Many techniques have been described for achieving this [15, $16,17,18]$. This paper describes the translation of CBCL and its adaptation to Sinhala.

\section{Method}

Translation/back-translation method was used in this endeavour, where the second author and two independent bilingual experts were involved. Final Sinhala translation was back-translated to English by a third bi-lingual expert who was not aware of the English version. Consensus regarding the preservation of original meaning of items of the CBCL was further checked by 4 mental health professionals.

To check content, semantics, and conceptual validity, a list of relevant questions regarding the translation was circulated among 6 mental health professionals independent of those involved in the translation, along with the original English version of CBCL, the Sinhala translation of CBCL (CBCL-S), and a rating scale. The questions focused on the following.
1. Whether each item is suitable to measure mental health in children aged 5-10 years.

2. Whether the Sinhala translation has the equivalent meaning of the English version.

3. Whether individual items in the translation are culturally appropriate to Sri Lanka.

4. Whether each item is an appropriate indicator of behaviour measured by the subscale in which it is included.

5. Whether items in a given subscale collectively measure that subscale adequately.

Responses for these questions were rated in a scale of 0-9, 0 indicating total disagreement and 9 indicating total agreement. A rating of 7 or above by $70 \%$ of the respondents was considered as satisfactory for each component rated $[18,19]$.

CBCL-S was pre-tested and piloted among primary carers of two groups of children selected from specialist child mental health clinics and from the community. Criterion validity of CBCL-S was established by using the clinical diagnosis of a specialist psychiatrist as the gold standard. For this purpose, 2 samples of children were used from specialist mental health clinics and the community.

Based on the expected sensitivity and specificity of the instrument, sample size required for validation was calculated as 49 boys and 49 girls with mental health problems and a similar number without mental health problems. Administration of CBCL-S to the primary carers of selected children was carried out by the first author or a trained research assistant.

Total scores obtained from CBCL-S in the above sample were graphed against clinical diagnoses to obtain receiver operator characteristics (ROC) curves for girls and boys separately. These were used to decide the appropriate cut-off levels of total scores.

Internal consistency of CBCL-S was measured by calculating Cronbach's alpha, using the same data that were used for validation of CBCL-S. To determine the testretest reliability, CBCL-S was first administered to 20 parents and re-administered after 2 weeks, and measured using Kappa. Inter-rater reliability was measured by readministering the instrument by the first author to 20 respondents who were initially seen by the research assistant.

\section{Results}

All 120 items were rated as appropriate for measuring mental health status of children aged 5-10 years. All raters rated 98 of the items as having correct translations; one respondent rated 22 items as " 6 " in the scale. While all of respondents rated over "7" for 118 items as culturally 
relevant, one respondent rated 2 items at "6". All respondents agreed that each item is an appropriate indicator of the behaviour measured by the subscale in which it is included and that the items in a given subscale collectively measure the subscale adequately.

Sixty-nine boys and 69 girls from the community and 80 boys and 49 girls from psychiatry clinics were assessed to measure criterion validity. Of them, 63 girls and 59 boys were clinically normal while 55 girls and 90 boys were clinically abnormal. Mean ages of girls with and without clinical problems were $8(\mathrm{SD}=1.73)$ and $7.7(\mathrm{SD}=1.33)$ respectively. For boys the mean ages were $8.1(\mathrm{SD}=1.55)$ and $7.9(\mathrm{SD}=1.32)$ respectively.

ROC curves were drawn separately for girls and boys. Area under the ROC curve was 0.95 for both boys (95\% CI: 0.92-0.98) and girls (95\% CI: 0.94-0.98). Cut-off level of 39 for CBCL-S total scores offered the maximum sensitivity and specificity for boys, which were $90 \%$ and $88 \%$ respectively. For girls, maximum sensitivity and specificity, which were $89 \%$ and $92 \%$ respectively, were offered at the same cut-off level of 39 .

Cronbach's alpha for internal consistency was estimated for each of CBCL-S scales (for boys and girls separately) and shown in table 1 . Table 2 shows crossscale correlation of individual symptom scales with total problems scale and internalising and externalising scales.

Paired-t test was performed for total CBCL-S scores obtained from test and re-test procedures. This showed that results of the two tests did not differ significantly. Intra-class correlation coefficient (ICC) calculated was 0.8 (95\% CI: 0.79-0.85). Total CBCL-S scores were then categorised in to dichotomous variables (normal and abnormal) using the cut-off point of 39. Cohen's kappa estimated using these dichotomised categories was 0.86 . Paired t-test for the inter-interviewer reliability showed that total scores obtained by the two interviewers did not differ significantly. These total CBCL-S scores were then dichotomised into normal and abnormal categories using the cut-off point of 39. Cohen's kappa that was estimated (0.78) showed good agreement between the results obtained by the two interviewers.

\section{Discussion}

Recommended guidelines for validating cross-cultural assessment tools by Guiliman [17] were followed in translating and adapting CBCL to Sinhala. Content, semantic, and conceptual validity were addressed during translation. In addition, the use of a panel of judges [18] to rate $\mathrm{CBCL}$ facilitated the process of obtaining satisfactory consensus on the above mentioned validity types. The translation procedure was similar to that employed in previous studies [20].

CBCL-S compares well with the original English CBCL and its translated versions for validity, reliability, and discriminatory ability. Areas under the ROC curves indicated that CBCL-S was superior to other Asian translations [20] in differentiating between boys and girls aged 5-10 years with and without behaviour problems.

Cut-off level for both girls and boys was taken separately as 39 . These cut-off points would ensure that there will be a minimal number of false positives and false negatives among the children researched using the CBCL-S. These cut-off levels are less than that for the original English CBCL [4]. It is not unusual for the cut-off

Table 1. Internal consistency of the CBCL-S scales

\begin{tabular}{lcc}
\hline \multirow{2}{*}{ Scale } & \multicolumn{2}{c}{ Alpha } \\
\cline { 2 - 3 } & Girls & Boys \\
\hline Total score & 0.73 & 0.74 \\
Internalising & 0.69 & 0.69 \\
Externalising & 0.79 & 0.79 \\
Withdrawn & 0.67 & 0.66 \\
Somatic complaints & 0.63 & 0.68 \\
Anxious / depressed & 0.69 & 0.70 \\
Social problems & 0.72 & 0.71 \\
Thought problems & 0.63 & 0.66 \\
Attention problems & 0.74 & 0.74 \\
Delinquent behaviour & 0.68 & 0.66 \\
Aggressive behaviour & 0.73 & 0.74 \\
\hline
\end{tabular}




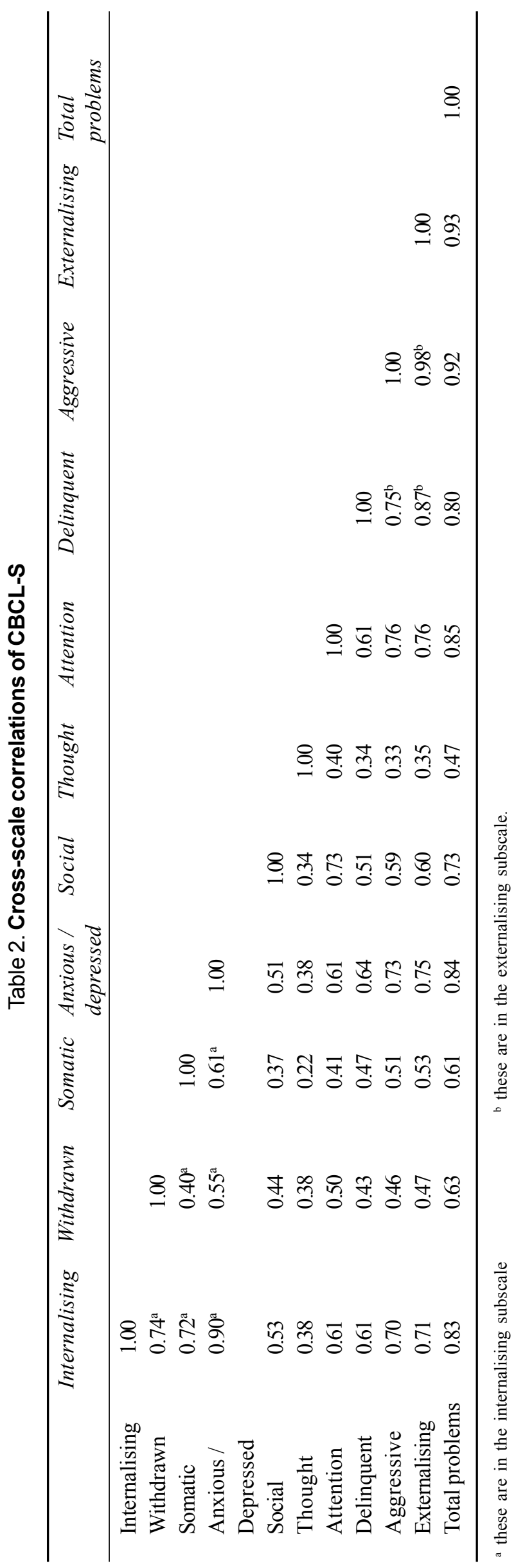

level of an instrument to vary from that of the original instrument depending on the cultural setting in which the translated instrument is used [18]. Indian and Chinese versions of the $\mathrm{CBCL}$ also have arrived at lower cut-off points than the English CBCL $[10,20]$.

At the cut-off level of 39 , the CBCL-S had a sensitivity of $90 \%$ and specificity of $88 \%$ for boys, and for girls a sensitivity of $89 \%$ and specificity of $92 \%$. These observations are similar to the findings of other validation studies of CBCL [5-10]. Specificity of CBCL-S was similar to that of Chinese CBCL but higher than the Indian CBCL. Sensitivity of CBCL-S is higher than both the Indian and Chinese versions $[10,20]$. Of the girls who scored over 39 , $91 \%$ were found to be clinically abnormal while of the girls who scored at lower levels, $91 \%$ were clinically normal. These figures were $92 \%$ and $85 \%$ respectively for boys.

Although the internal consistency for the total problem score is less than that for the original English CBCL [4], which was 0.9 , Cronbach's alpha of 0.7 can be considered as a satisfactory level of reliability.

In the cross-scale correlation, all subscales that form the internalising scale (withdrawn, somatic complaints, and anxious / depression) have good positive correlation with the internalising scale. Similarly, those subscales that form the externalising scale (delinquent behaviour and aggressive behaviour) have good positive correlation with the externalising scale. A low correlation was seen between total problem score and the somatic, anxious/depression, withdrawn and thought problems subscale. This exception could be based on Sri Lankan children having more externalising problems rather than internalising problems. Another possibility is that unlike depressed and anxious adults, children with similar emotional problems present more with problem behaviours such as irritability, restlessness, and temper tantrums. Furthermore, parents' ignorance about minor behaviour changes in the child could be a reason for the thought problems to have a low correlation with the total problem scores. Except the withdrawn and somatic scales, which showed moderately positive correlation with the total problem scores, all other scales showed good positive correlation. This further supports the good internal consistency of CBCL-S.

There was no significant difference between total problem scores obtained during test-retest procedure. ICC exceeded the minimum satisfactory level of 0.7 [18]. English CBCL validation had an ICC of 0.9 for test-retest procedure [4] and the Chinese CBCL had ICC of 0.8 [20]. Cohen's kappa of over 0.8 for test-retest procedure is considered satisfactory and is better than what was found in other Asian countries [20]. Cohen's kappa of 0.8 for the inter-interviewer reliability was satisfactory, which is similar to inter-interviewer reliability of the original American CBCL [4].

None of the subscales of CBCL-S was validated due to practical limitations. Hence, no cut-off levels are identifiable for different mental health syndrome scales in 
CBCL-S. However, the overall results indicate that CBCL$\mathrm{S}$ is a valid and reliable instrument to measure mental health status of children aged 5-10 years and justify application in clinical and research settings. The users must be warned about the practical difficulties that may arise due to the length of the instrument. Also, caution should be advocated regarding cut-off levels to be used in widely contrasting populations of children.

\section{Acknowledgements}

We thank Prof. Kumudu Wijewardene (Dean / Faculty of Graduate Studies, University of Sri Jayewardenepura), Sida / SAREC grant for 'Health and Social Care for Socially Marginalised People', staff of IMCH / University of Uppsala, and all healthcare professionals who participated in the research.

\section{References}

1. Child Behaviour Checklist (Online). (Cited 31 January 2005). Available from: URL: http://www.aseba.org/products/cbcl618.html.

2. Marydith SP. Social desirability and behaviour rating scales: an exploratory study with the child behaviour checklist/4-18. Psychology in the Schools 2003; 40: 225-5.

3. Crijnen AA, Achenbach TM, Verhulst FC. Problems reported by parents of children in multiple cultures: the child behaviour checklist syndrome constructs. American Journal of Psychiatry 1999; 156: 569-74.

4. Achenbach TM. Manual for the Child Behaviour Checklist/ 4-18 and 1991 Profile. Burlington, VT: University of Vermont Department of Psychiatry, 1991.

5. Rubio-Stipec M, Bird H, Canino G, Gauld M. The internal consistency and concurrent validity of a Spanish translation of the child behaviour checklist. Journal of Abnormal Child Psychology 1990; 18: 393-406.

6. Nelson EC, Hanna GL, Hudziak JJ, Botteron KN, Heath AC, Todd RD. Obsessive-compulsive scale of the child behaviour checklist: specificity, sensitivity, and predictive power. Paediatrics 2001; 108: 14-15.

7. Novik TS. child behaviour checklist item scores in Norwegian children. European Child and Adolescent Psychiatry 2000; 9: 54-60.

8. Kaisus MC, Ferdinand RF, van den Berg H, Verhulst FC. Associations between different diagnostic approaches for child and adolescent psychopathology. Journal of Child Psychology and Psychiatry 1997; 38: 625-32.

9. Leung PWL, Kwong SL, Tang CP, Ho TP, Hung SF, Lee CC, et al. Test-retest reliability and criterion validity of the Chinese version of CBCL, TRF, and YSR. Journal of Child Psychology and Psychiatry 2006; 47: 970-3.

10. Srinath S, Girimaji SC, Gururaj G, Seshadri S, Subbakrishna DK, Bhola P, et al. Epidemiological study of child and adolescent psychiatric disorders in urban and rural areas of Bangalore, India. Indian Journal of Medical Research 2005; 122: $67-79$.

11. Chang AM, Chau JPC, Holroid E. Translations of questionnaires and issues of equivalence. Journal of Advanced Nursing 1999; 29: 316-22.

12. International Test Commission. ICT Guidelines on adapting tests, translation and test use (Online). (Cited 20th February 2005). Available from: URL: http://www.intestcom.org

13. Brazier JE, Harper R, Jones NMB, et al. Validating the SF36 Health survey questionnaire: new outcome measure for primary care. British Medical Journal 1992; 305: 160-4.

14. Garrat AM, Ruta DR, Abdulla MI, et al. The SF-36 Health survey questionnaire: an outcome measure suitable for routine use within the NHS? British Medical Journal 1993; 306: $1440-4$.

15. Prieto AJ. A method for translation of instruments to other languages. Adult Education Quarterly 1992; 43: 1-14.

16. Sumathipala A, Murray J. New approach to translating instruments for cross-cultural research: a combined qualitative and quantitative approach for translation and consensus generation. International Journal of Methods in Psychiatric Research 2000; 9: 87-95.

17. Guilliman F, Bombardier C, Beaton D. Cross cultural adaptation of health related quality of life measures: literature review of proposed guidelines. Journal of Clinical Epidemiology 1993; 46: 1417-32.

18. De Zoysa PT. Parental use of physical force towards 12year-old school children in the Colombo District: prevalence, psychosocial correlates, and psychological consequences. Thesis submitted to the University of Colombo for the degree of Doctor of Philosophy. 2006.

19. Fink A, Kosecoff J, Chassin M, Brook RH. Consensus methods, characteristics and guidelines for use. American Journal of Public Health 1984; 74 : 979-83.

20. Leung PWL, Kwong SL, Tang CP, Ho TP, Hung SF, Lee CC, et al. Test-retest reliability and criterion validity of the Chinese version of CBCL, TRF, and YSR. Journal of Child Psychology and Psychiatry 2006; 47: 970-3. 